\title{
Motion Analysis and Prediction Using Machine Learning Models and Fuzzy Logic
}

\author{
Vishesh S ${ }^{1}$, Anil Umesh ${ }^{2}$ \\ BE (TCE), MBA, PGDIB ${ }^{1}$ \\ Student, MS, Information Systems, University of Maryland, Baltimore County, Maryland, USA ${ }^{2}$
}

\begin{abstract}
With the ever increasing computational ability of modern machines and their ability to learn by themselves has led to the sustained growth of Artificial Intelligence. When measuring few parameters from the ether (dynamic), huge amount of data is obtained. The amount of knowledge available is too large for explicit encoding by humans. Machines that learn this knowledge can reproduce more accurate results/ predictions at specified instants. Environments change over time. Machines that can adapt to a changing environment would reduce the need for constant redesign. In this paper we obtain motion values of objects in a lab environment and make analysis of the dynamics using fuzzy logic. The machine is trained using machine learning algorithms to make predictions on motion. These predictions are further fed to the machine to increase the intelligence of the predicting model and to establish a controlled loopback model. Use of fuzzy logic, further refines the accuracy of the approach and rules out the present incompatible two logic level approach.
\end{abstract}

Keywords: Fuzzy Logic, Data Analytics and Machine Learning, Artificial Intelligence, Logistic regression, accuracy, precision, F1 score, categorical values, visualization, Heatmap analysis is done for knowledge of correlation

\section{INTRODUCTION}

There are some characteristic properties of data obtained from the Ether:

- Huge data sets,

- Heterogeneity, and

- Uncertainty, due to:

i. presence of random variables,

ii. incomplete data or inaccurate data (inaccuracy of measurement),

iii. approximate estimations instead of measurements (due to technical or financial problems),

iv. incomparability of data (varying measurement or observation conditions),

v. qualitative instead quantitative information (due to technical or financial problems),

vi. $\quad$ incomplete or vague expert knowledge, and

vii. $\quad$ Subjectivity of the information obtained from expert.

An AI system can be designed with certain entities which behave dynamically during changing sensory signals, goals and resulting actions. Any change in

- Perception

- Model

- Planning and reasoning

- Action computation

would be called learning. Different learning mechanism might be employed depending on which subsystem is being changed. Figure 1 shows the AI system and various objects/ entities.

\section{MOTIVATION}

Dynamic nature of motion of objects and the ever increasing randomness of the events has triggered a need to measure and calibrate certain actions with respect to time. When a proper relationship between input /output pair cannot be established, machines can be made to adjust their internal structure to produce correct outputs for a large number of sample inputs and thus suitably constrain their input /output function. 


\section{PROBLEM STATEMENT}

An array of ultrasonic sensors is placed at different orientations collecting data for a particular interval of time. The acquired sensor values are subjected to pre-processing, data analytics and visualization. The acquired data is made to correspond to a particular Fuzzy Logic by means of programming.The prepared data needs to be fed to the machine, for it to learn. Various graphical and analytical tools have to be incorporated to achieve a relation between the so considered independent and dependent column attributes.

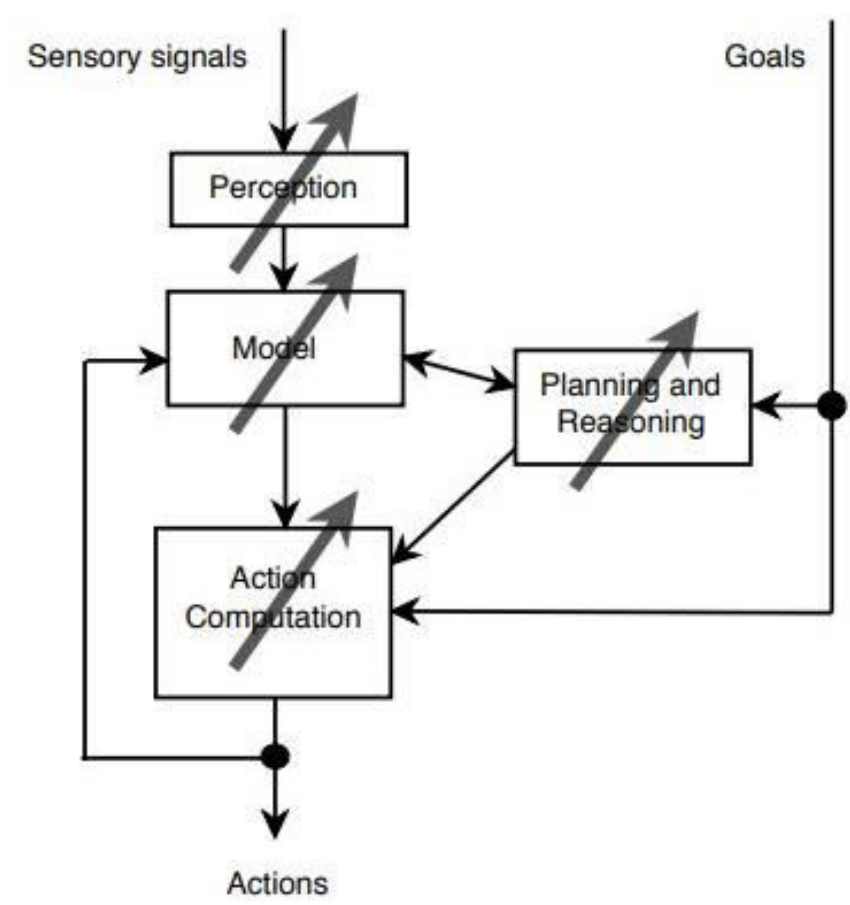

Figure 1 shows the AI system and various objects/ entities.

\section{BLOCK DIAGRAM}

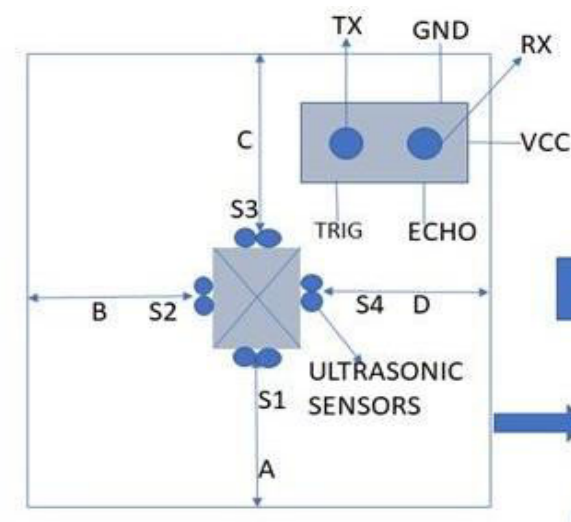

\section{MICROCONTROLLER}

DATA PROCESSING
$\&$
MACHINE LEARNING

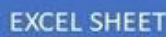

\begin{tabular}{|c|c|c|}
\hline $\begin{array}{c}\text { A-distance A } \\
\text { B-distance B } \\
\text { C-distance C } \\
\text { D-distance D }\end{array}$ & speed of sound=343m/s @25 ${ }^{\circ} \mathrm{C}$ & $\begin{array}{l}\text { S1-sensor } 1 \\
\text { S2-sensor 2 } \\
\text { S3-sensor 3 } \\
\text { S4-sensor 4 }\end{array}$ \\
\hline
\end{tabular}

Figure 2 shows the block diagram of the proposed system. 
Vol. 8, Issue 11, November 2019

Figure 2 shows the block diagram of the proposed system. The sensors S1, S2, S3 and S4 are placed in an array at different orientations. More sensors could be added to obtain better result. An ultrasonic sensor continuously records data and is moved to the database. The data can be pre-processed before storing or formatted after, on the excel sheet.

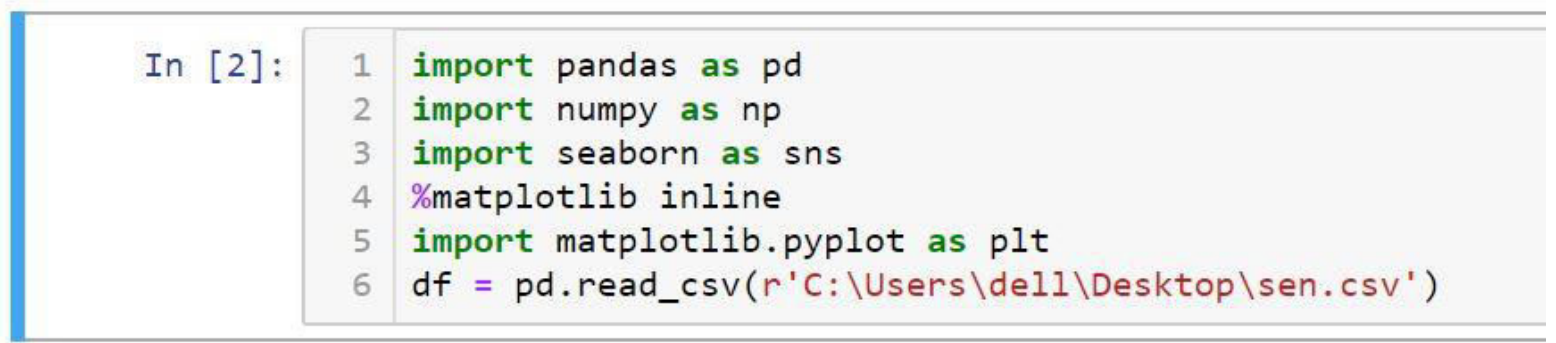

Figure 3 shows the Python code to import libraries.

\section{METHODOLOGY}

The work process can be achieved in the following steps

i. Data acquisition from the sensor array and storing in the database. Figure 3 shows the Python code to import libraries. [2]

ii. The data may contain noise, NaN values, and information that should be treated before analysis or visualization. This is called data pre-processing. Figure 4 shows the dataset before pre-processing and removal of $\mathrm{NaN}$ values. [3]

iii. The pre-processed data is now eligible for data analytics and data visualization. Graphs and statistical formulas enhance the process of AI.

\begin{tabular}{|c|c|c|c|c|c|c|c|c|}
\hline & TIME & DISTANCEA & DISTANCEB & FUZZA & CATA & CATB & FUZZB & FOR \\
\hline 0 & 0 & 48.0 & 80.0 & 1.020 & 1.0 & 0.0 & $\mathrm{NaN}$ & 1.0 \\
\hline 1 & 1 & 7.0 & 50.0 & 1.020 & 4.0 & 0.0 & $\mathrm{NaN}$ & 1.0 \\
\hline 2 & 2 & 62.0 & 80.0 & 1.010 & 2.0 & 3.0 & 1.0 & 1.0 \\
\hline 3 & 3 & 48.0 & 70.0 & 1.005 & 4.0 & 0.0 & 1.0 & 0.0 \\
\hline 4 & 4 & 51.0 & 80.0 & 1.010 & 2.0 & 0.0 & 1.0 & 1.0 \\
\hline 5 & 5 & 60.0 & 90.0 & 1.015 & 3.0 & 0.0 & $\mathrm{NaN}$ & $\mathrm{NaN}$ \\
\hline 6 & 6 & 68.0 & 70.0 & 1.010 & 0.0 & 0.0 & $\mathrm{NaN}$ & 1.0 \\
\hline 7 & 7 & 24.0 & $\mathrm{NaN}$ & 1.015 & 2.0 & 4.0 & 1.0 & 0.0 \\
\hline 8 & 8 & 52.0 & 100.0 & 1.015 & 3.0 & 0.0 & 1.0 & 0.0 \\
\hline 9 & 9 & 53.0 & 90.0 & 1.020 & 2.0 & 0.0 & 0.0 & 0.0 \\
\hline 10 & 10 & 50.0 & 60.0 & 1.010 & 2.0 & 4.0 & $\mathrm{NaN}$ & 0.0 \\
\hline 11 & 11 & 63.0 & 70.0 & 1.010 & 3.0 & 0.0 & 0.0 & 0.0 \\
\hline 12 & 12 & 68.0 & 70.0 & 1.015 & 3.0 & 1.0 & $\mathrm{NaN}$ & 1.0 \\
\hline 13 & 13 & 68.0 & 70.0 & $\mathrm{NaN}$ & $\mathrm{NaN}$ & $\mathrm{NaN}$ & $\mathrm{NaN}$ & $\mathrm{NaN}$ \\
\hline 14 & 14 & 68.0 & 80.0 & 1.010 & 3.0 & 2.0 & 1.0 & 0.0 \\
\hline 15 & 15 & 40.0 & 80.0 & 1.015 & 3.0 & 0.0 & $\mathrm{NaN}$ & 1.0 \\
\hline
\end{tabular}

Figure 4 shows the dataset before pre-processing and removal of $\mathrm{NaN}$ values. 
Vol. 8, Issue 11, November 2019

iv. The data-set is now made into a Fuzzy logic as shown in figure 5.

v. Data visualization is carried out on the formatted data set. Figure 6 shows the graph of fuzzy logic count. Figure 7 shows a line graph of fuzz vs. format.

vi. Heatmap analysis is done for knowledge of correlation. The data is split into train and test using a particular randomness factor ' $\mathrm{X}$ '. [4] Figure 8 shows the heatmap of the assignment.

vii. A predictive model is designed using Logistic regression and its accuracy is verified. [5]. Figure 9 shows the result with accuracy.

\begin{tabular}{|c|c|c|c|c|c|c|c|c|}
\hline \multirow[t]{12}{*}{ out [3]: } & & current time & Distance in $\mathrm{cm}$ & distaA in $\mathrm{cm}$ & formatA & format & fuzzA & fuzz \\
\hline & 0 & $12: 13: 11$ & 200.00 & 26.10 & 1 & 0 & 0.20 & 0.0 \\
\hline & 1 & $12: 13: 11$ & 8.45 & 27.75 & 1 & 1 & 0.20 & 0.1 \\
\hline & 2 & $12: 13: 12$ & 8.35 & 12.61 & 1 & 1 & 0.10 & 0.1 \\
\hline & 3 & $12: 13: 12$ & 9.76 & 12.19 & 1 & 1 & 0.10 & 0.1 \\
\hline & 4 & $12: 13: 12$ & 8.35 & 12.62 & 1 & 1 & 0.10 & 0.1 \\
\hline & 5 & $12: 13: 12$ & 8.45 & 13.89 & 1 & 1 & 0.10 & 0.1 \\
\hline & 6 & $12: 13: 12$ & 8.35 & 12.18 & 1 & 1 & 0.10 & 0.1 \\
\hline & 7 & $12: 13: 13$ & 8.47 & 12.61 & 1 & 1 & 0.10 & 0.1 \\
\hline & 8 & $12: 13: 13$ & 8.33 & 41.93 & 1 & 1 & 0.20 & 0.1 \\
\hline & 9 & $12: 13: 13$ & 8.76 & 10.07 & 1 & 1 & 0.10 & 0.1 \\
\hline & 10 & $12: 13: 13$ & 9.76 & 10.08 & 1 & 1 & 0.10 & 0.1 \\
\hline
\end{tabular}

Figure 5 shows Fuzzy logic implementation.

$f=$ plt.subplots $($ figsize $=(22,5))$

color_types =['\#FFO000', '\#00000F', '\#AAAAAA', '\#00FF00']

sns.countplot $(x="$ fuzzA", palette $=$ color_types, data $=d f)$.set_title( 'chem pass' $)$;

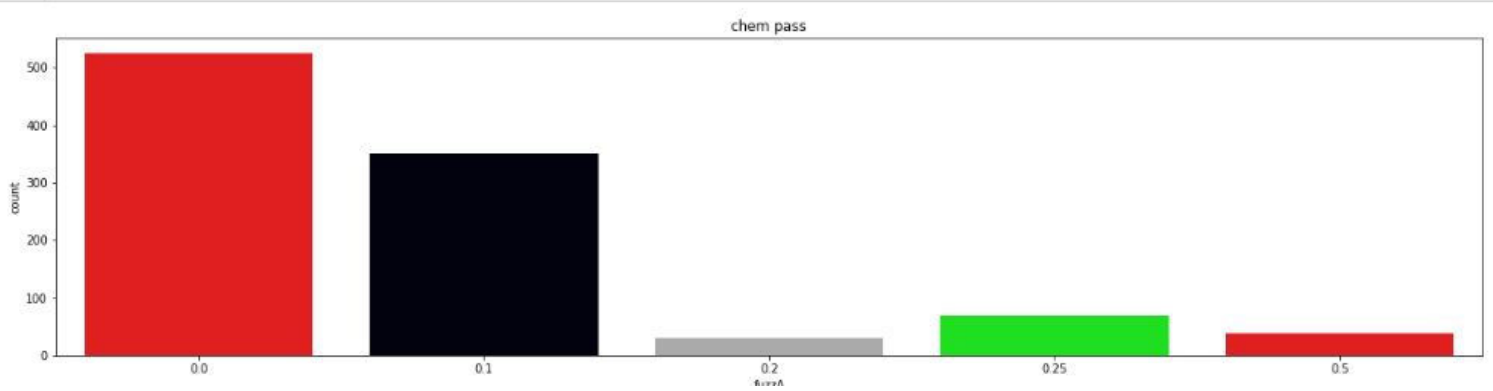

Figure 6 shows the graph of fuzzy logic count.

ax=sns. lineplot ( $x=$ "fuzzA", $y="$ formatA", data=df)

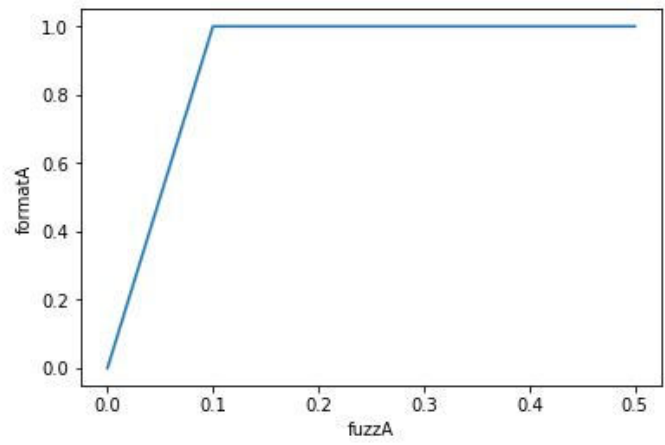

Figure 7 shows a line graph of fuzz vs. format. 
International Journal of Advanced Research in Computer and Communication Engineering

Vol. 8, Issue 11, November 2019

\begin{tabular}{rrrrrrr} 
& Distance in cm & distaA in cm & formatA & format & fuzzA & fuzz \\
\hline Distance in cm & 1.000000 & -0.018671 & 0.028335 & -0.978855 & 0.023904 & -0.360664 \\
distaA in cm & -0.018671 & 1.000000 & -0.570074 & 0.018835 & -0.382703 & 0.020258 \\
formatA & 0.028335 & -0.570074 & 1.000000 & -0.032605 & 0.709676 & 0.001149 \\
format & -0.978855 & 0.018835 & -0.032605 & 1.000000 & -0.034827 & 0.501482 \\
fuzzA & 0.023904 & -0.382703 & 0.709676 & -0.034827 & 1.000000 & -0.021508 \\
fuzz & -0.360664 & 0.020258 & 0.001149 & 0.501482 & -0.021508 & 1.000000
\end{tabular}

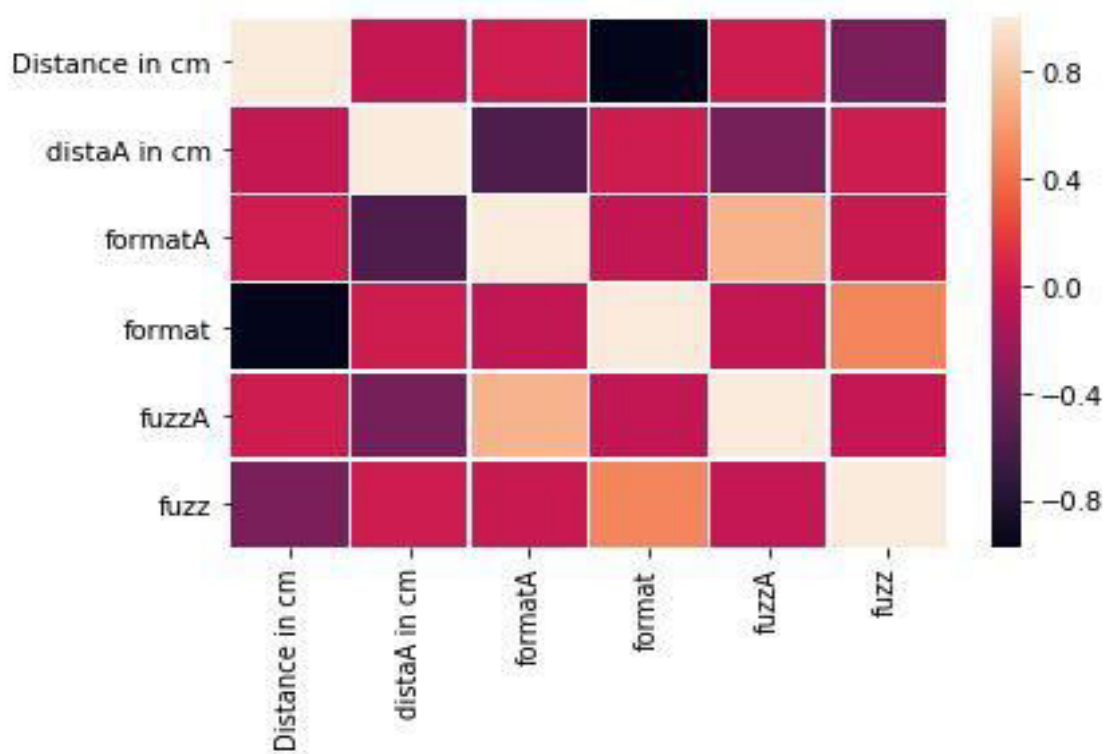

Figure 8 shows the heatmap of the assignment.

In $[86]$ :

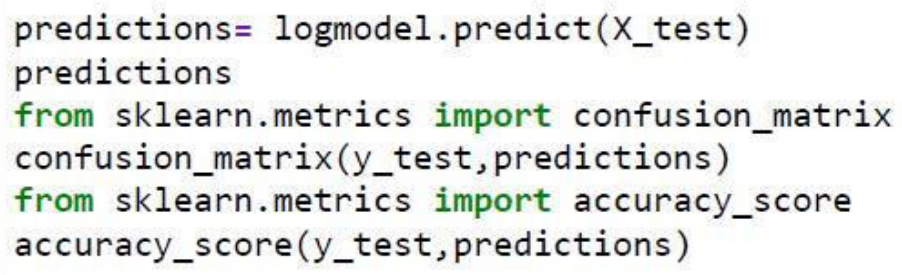

0.9833333333333333

Figure 9 shows the result with accuracy.

\section{CONCLUSIONS}

Motion detection is a simple task because of the improvement in the sensor technology and with ever increasing accuracy of the same. The major challenge that we have encountered is the prediction of motion of any object with the existing data and runtime data. This can be done by Machine Learning algorithms which allow the machines to learn on their own. Logistic regression is one such Machine Learning algorithm used to model the probabilities for classification problems with two possible outcomes. Ultrasonic sensors were mounted at different orientations, continuously gathering data of the ether. The data acquired is then analysed, visualized and used for future predictions/ conclusions. Figure 10 shows the predictions of the project. 


\begin{tabular}{|c|c|c|}
\hline & 1 & predictions \\
\hline & & 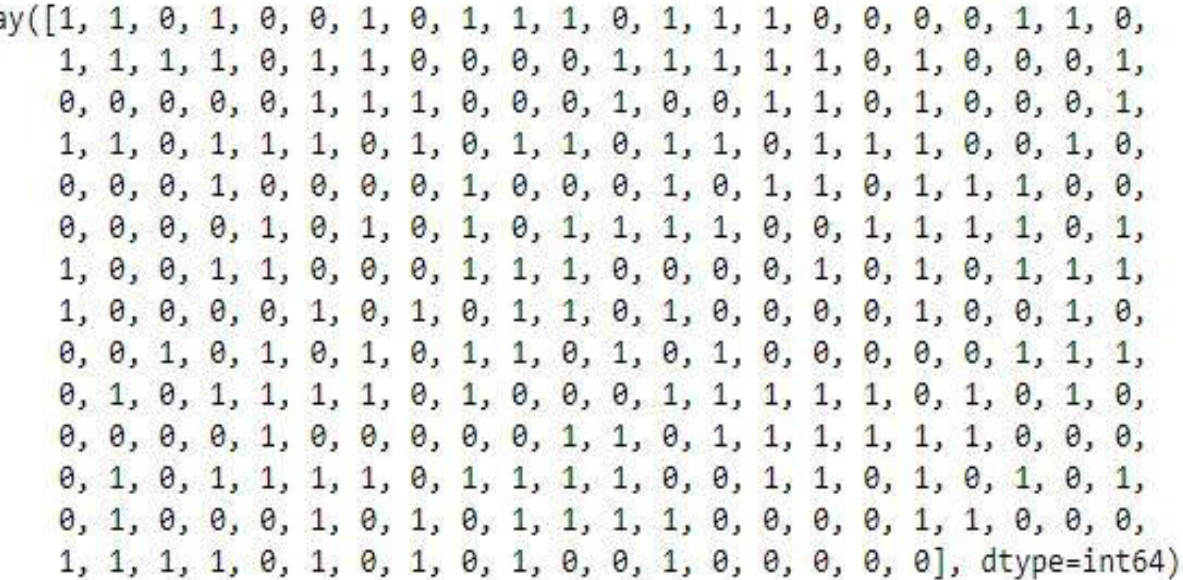 \\
\hline
\end{tabular}

Figure 10 shows the predictions of the project.

\section{REFERENCES}

[1]. Moving Object Tracking Method Based on Ultrasonic Automatic Detection Algorithm- Damei Fu and Zhihong Zhao- 2016- IEEE.

[2]. The Python Standard Library — Python 3.7.1rc2 documentation https://docs.python.org/3/library/

[3]. Data Warehousing Architecture and Pre-Processing- Vishesh S, Manu Srinath, Akshatha C Kumar, Nandan A.S. - IJARCCE, vol 6, issue 5, May 2017.

[4]. Data Mining and Analytics: A Proactive Model - http://www.ijarcce.com/upload/2017/february-17/IJARCCE\%20117.pdf

[5]. A comparative analysis on linear regression and support vector regression- DOI: 10.1109/GET.2016.7916627- https://ieeexplore.ieee.org /abstract/document/7916627

\section{BIOGRAPHY}

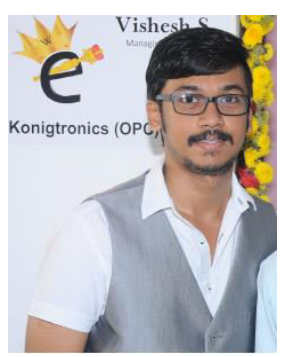

VISHESH S (BE, MBA, PGDIB, (MTech)) born on $13^{\text {th }}$ June 1992 hails from Bangalore (Karnataka) and has completed B.E in Telecommunication Engineering from VTU, Belgaum, Karnataka in 2015. He also worked as an intern under Dr. Shivananju BN, former Research Scholar, Department of Instrumentation, IISc, Bangalore. His research interests include Embedded Systems, Wireless Communication, BAN and Medical Electronics. He is also the Founder and Managing Director of the corporate company Konigtronics Private Limited. He has guided over a thousand students/interns/professionals in their research work and projects. He is also the co-author of many International Research Papers. He has recently completed his MBA in e-Business and PG Diploma in International Business. Presently Konigtronics Private Limited has extended its services in the field of Software Engineering and Webpage Designing. Konigtronics also conducts technical and non-technical workshops on various topics. Real estate activities are also carried out under the guidance of Siddesh B S BE (civil). Vishesh S along with his father BS Siddesh has received various awards and applauses from the scientific and entrepreneurial society. He was appointed as the MD of Konigtronics Pvt Ltd (INC. on ${ }^{\text {th }}$ Jan 2017) at an age of 23 years. His name is indexed in various leading newspapers, magazines, scientific journals and leading websites \& entrepreneurial forums. He is also the guide for many international students pursuing their Masters. 